\title{
La Educación de Calidad para Todos Eupieza en la Primera Infancia
}

\author{
Education of Quality for all Starts in the Early Infancy
}

\author{
María Rosa Blanco Guijarro \\ Especialista en Educación Diferencial e Inicial \\ UNESCO \\ Enrique Delpiano 2058, Providencia, Santiago \\ E-mail: rblanco@unesco.cl
}

Resumen: Se postula que la educación de calidad para todos implica el desarrollo de tres elementos que le son esenciales: pertinencia y relevancia, equidad y protección de los derechos humanos de la persona. Se abordan, también, temas relacionados con el aporte que haría la educación de la primera infancia a la calidad de la misma y los criterios que regináan para definir dicha calidad en menores de seis años, debido a sus caracteństicas y necesidades. Se presentan, además, diversos aspectos que conducináan a la calidad educacional deseada.

Palabras clave: educación de calidad, primera infancia, pertinencia y relevancia, equidad, derechos humanos.

Abstract: It is postulated that education of quality for all implies the development of three elements which are essential: pertinency and relevance; equality; and preservation of the human rights of the individual. It enters upon matters referring to the contribution of education in early infancy to its quality and the criteria which would stand on the definition of such quality, with reference to infants under six years, due to their characteristics and needs. Different aspects that would lead to the desired quality are presented.

Key words: education of quality, infancy, pertinency and relevance, equality, human rights.

\section{INTRODUCCIÓN}

El presente artículo está organizado en torno a tres preguntas. En un primer momento, de forma muy sucinta, se aportan algunos elementos que tratan de dar respuesta a una pregunta que se hacen investigadores, tomadores de decisiones, docentes y familias; ¿qué es una educación de calidad para todos? Se desarrollan tres elementos que actualmente son esenciales para definir una educación de calidad: que satisfaga los derechos de las personas, que sea pertinente y relevante y que sea equitativa.

La segunda pregunta que se aborda es jpuede la educación de la primera infancia hacer la diferencia en lograr una educación de calidad para todos? Para dar respuesta a la misma se utilizan diferentes argumentos que muestran como la educación y cuidado de la primera infancia marcan la diferencia en el desarrollo de las personas y en el conjunto de las sociedades. 
La tercera cuestión que se aborda es cexisten criterios específicos para definir la calidad de la educación de la primera infancia? Se parte de la base de que las características que definen una educación de calidad en general también son válidas para la educación inicial, sin embargo, además existen algunos elementos propios de esta etapa por las características y necesidades de los menores de seis años. El artículo finaliza presentando algunos desafíos que es preciso enfrentar para avanzar hacia una educación de mayor calidad en la primera infancia.

\section{1. ¿QUÉ ES UNA EDUCACIÓN DE CALIDAD PARA TODOS?}

El derecho a la educación como un derecho básico humano se estableció en la Declaración Universal de Derechos Humanos (1948), y ha sido reafirmado en la Convención sobre los Derechos del Niño, la cual ha sido ratificada casi universalmente. Sin embargo, todavía existen en el mundo millones de personas para las cuales no se ha hecho efectivo este derecho. Por este motivo, tuvo lugar la Conferencia Mundial de Educación para Todos (Jomtien 1990) (UNESCO 1990), en la que por primera vez los dirigentes mundiales comienzan a enfrentar el desafío de la lucha contra la exclusión.

En esa oportunidad se establecieron varios objetivos orientados a universalizar la educación primaria, aumentar el acceso a la educación de la primera infancia, reducir el analfabetismo y mejorar la calidad. Una de las recomendaciones básicas de esta Conferencia fue la de fomentar la equidad, tomando medidas sistemáticas para reducir las desigualdades y suprimir las discriminaciones referidas a las posibilidades de aprendizaje de los grupos en situación de desventaja.

La evaluación de los diez años de Educación para Todos puso de manifiesto que, a pesar de los esfuerzos realizados por los países, los avances habían sido muy insuficientes por lo que en el Foro Mundial de Educación para Todos (Dakar 2000) (UNESCO, 2000), los países reafirmaron su compromiso con la Declaración Mundial sobre Educación para Todos de Jomtien.

En los marcos de acción de Educación para Todos planteados tanto en Jontiem (1990) como en Dakar (2000) se considera que la calidad de la educación es fundamental para lograr la educación para todos. En el marco de acción de Dakar, el objetivo 6 está referido explícitamente a la calidad y también se alude a ella en los objetivos relacionados con la universalización de la educación primaria y el aumento de la expansión de la educación de la primera infancia.

Lograr una educación de mayor calidad no es sólo un acuerdo internacional sino que es una de las principales aspiraciones de los países, sin embargo, primero suele enfrentarse el objetivo del acceso universal a la educación y 
luego se piensa en la calidad de la misma, cuando son dos aspectos estrechamente relacionados. Una educación de calidad marca la diferencia en los resultados de aprendizaje de los alumnos y en los niveles de asistencia y finalización de estudios, por lo que finalmente la calidad de la educación influye en su expansión.

El concepto de calidad no es unívoco sino que, por el contrario, existen diferentes visiones y enfoques, dependiendo de distintos factores tales como las funciones que se asignen a la educación en un momento y contexto determinado, las concepciones sobre el aprendizaje y la enseñanza, o las exigencias de la sociedad, por nombrar algunos de ellos. No obstante, cada vez existe mayor acuerdo respecto a que los resultados que logran los alumnos en determinadas áreas de aprendizaje, especialmente lenguaje y matemáticas, no es un elemento suficiente para definir la calidad de la educación, aunque sea lo que suele medirse en la mayoría de los países.

En el último informe de monitoreo de Educación para Todos, cuyo foco ha sido precisamente la calidad, se señalan tres aspectos en los que actualmente existe mayor consenso: la necesidad de respetar los derechos de las personas, la necesidad de una mayor equidad en el acceso, procesos y resultados, y la necesidad de una mayor pertinencia (UNESCO, 2005). A estos aspectos habría que añadir, también, el de la relevancia.

\subsection{Pertinencia y relevancia de la educación}

Una primera cuestión para valorar si una educación es de calidad es si ésta es coherente y cumple con los fines y funciones que se le asignan, los cuales varían con el tiempo y de un contexto a otro.

Actualmente pareciera existir cierto consenso respecto a que una de las finalidades más importantes de la educación es promover el desarrollo integral de las personas para que puedan ser miembros activos en la sociedad y ejercer la ciudadanía. La educación no sólo es un elemento clave para el desarrollo de las personas sino también para el de las sociedades, por ello se considera como uno de los elementos clave en el Índicededesarrollo humano.

El desarrollo humano, tanto a nivel individual como social, involucra dos procesos simultáneos que han de promoverse a través de la educación: la socialización y la individuación.

La socialización implica que las nuevas generaciones se apropien de los contenidos de la cultura y adquieran las competencias necesarias para ser miembros activos en dicha cultura. La inclusión y participación en la sociedad y el pleno ejercicio de la ciudadanía dependen en la actualidad de una serie de conocimientos y habilidades que no están igualmente distribuidos en la sociedad. 
La individuación es la construcción de cada persona como sujeto en el contexto de la sociedad en la que está inmerso, lo que significa promover la autonomía, la creatividad y la libre elección; en definitiva, la realización personal y la construcción de un proyecto de vida. La educación tiene como finalidad promover crecientes niveles de autonomía y de autogobierno.

Aprender a vivir juntos y Aprender a emprender. El aprendizaje de estos pilares ha de comenzar desde el nacimiento y continuar a lo largo de la vida.

Pero, además, una educación es de calidad si es pertinente y significativa para las personas de distintos estratos sociales y culturas, y con diferentes talentos, de forma que puedan construirse como sujetos en la sociedad y desarrollar su propia identidad. Las personas tienen múltiples inteligencias y distintos modos de aproximarse a la realidad, por lo que la educación ha de ofrecer diferentes opciones que permitan el desarrollo de distintas capacidades e intereses.

El tipo de normas y valores que existen en la escuela es uno de los factores que más influyen en la atención de la diversidad y en la construcción de la identidad personal y cultural. Los objetivos, las normas y experiencias que se brindan en la escuela, pueden no ser significativos y adecuados para muchos alumnos, e incluso ser contradictorios con sus experiencias previas. Esta situación incrementa la distancia entre los intereses de los alumnos y de la escuela, lo que repercute en sus progresos educativos. Los alumnos que provienen de contextos de menores recursos, que pertenecen a o otras culturas, o que tienen dificultades de aprendizaje y de participación no se sienten capaces de enfrentar con éxito las tareas escolares, lo que conlleva una falta de motivación y de esfuerzo que repercute en sus logros educativos.

\subsection{Calidad de educación para todos: un asunto de derechos humanos}

La Educación es un bien específicamente humano que surge de la necesidad de desarrollarse como tal, por ello todas las personas, sin excepción, tienen derecho a ella. Es decir, la educación permite la humanización. Tal como lo expresa Mantovani (1957), el hombre es la única criatura capaz de ser educada. El hombre puede ser hombre sólo mediante la educación.

El derecho a la educación no significa sólo acceder a ella sino también que ésta sea de calidad y logre que los alumnos aprendan lo máximo posible; el derecho a la educación es también el derecho a aprender y a desarrollar los múltiples talentos y capacidades de cada persona.

La enorme importancia del derecho a la educación radica en que, gracias a ella, es posible hacer efectivos otros derechos individuales y políticos y, en consecuencia, ejercer plenamente la ciudadanía, lo cual es el fundamento 
de una sociedad más justa y democrática. Para que ello sea posible es necesario "educar en y para los derechos humanos".

La educación para los derechos humanos forma parte del derecho a la educación y es un componente esencial de una educación de calidad. Educar en derechos humanos implica el aprendizaje no sólo de conocimientos y habilidades sino sobre todo de valores, actitudes y comportamientos. En este sentido, la educación en derechos humanos ha de estar presente en cualquier contexto y proceso educativo e involucra al conjunto del sistema educativo.

Así como la educación hace posible el ejencicio de otros derechos, es importante asegurar otros derechos para hacer plenamente efectivo el derecho a la educación, como son el derecho a la participación y a la no discriminación y a la propia identidad.

El derecho a la participación y a la no discriminación significa que ninguna persona debería sufrir ningún tipo de discriminación para participar en las diferentes actividades de la vida humana. En el ámbito educativo quiere decir que todos los niños y todas las niñas deberían educarse juntos en la escuela de su comunidad, independientemente de cual sea su origen social y cultural y sus características personales. Desde esta perspectiva, la escolarización en escuelas, grupos especiales o programas especiales, con carácter permanente, debería ser una excepción, y habría que asegurar que la enseñanza que se ofrezca equivalga al currículo común tanto como sea posible.

Lograr la individuación del sujeto, que es una de las finalidades de la educación, requiere asegurar el derecho a la propia identidad. Este supone un conjunto de atributos, de cualidades, tanto de carácter biológico como los referidos a la personalidad, que permiten precisamente la individuación de un sujeto en la sociedad. Atributos que facilitan decir que cada uno es el que es y no otro.

Se trata, en definitiva, que tanto la persona como la sociedad asuman la singularidad de cada ser humano y se valoren positivamente las diferencias, ya que éstas enriquecen a las personas y a la sociedad en su conjunto. La valoración negativa de las diferencias conlleva a la exclusión y la discriminación.

\subsection{Calidad y equidad de la educación: dos caras de la misma moneda}

Lograr una educación de calidad para todos, que promueva el máximo desarrollo, aprendizaje y participación de cada niño y niña, sólo será posible si se asegura el principio de igualdad de oportunidades; es decir, proporcionar a cada quien lo que necesita en función de sus características y necesidades individuales. 
La equidad significa asegurar la igualdad de oportunidades no sólo en el acceso a la educación, sino también en los años de estudios, en la calidad de la oferta y de los procesos educativos, en los aprendizajes que alcanzan los alumnos, y en el acceso a las tecnologías de la información y comunicación, con el fin de cerrar la brecha digital.

Si bien la educación por si sola no puede compensar las desigualdades sociales -es necesario también desarrollar políticas económicas y socialesconstituye una herramienta fundamental para acceder a empleos más productivos y para lograr la movilidad social. Hoy en día la escuela no es ni mucho menos el único espacio para acceder al conocimiento, pero todavía es la única instancia que asegura una distribución equitativa del mismo.

América Latina se caracteriza por ser la región más desigual del mundo. Las sociedades son altamente desintegradas y fragmentadas debido a la persistencia de la pobreza y la gran desigualdad en la distribución de los ingresos, lo cual genera altos índices de exclusión. Todos los países vienen realizando importantes esfuerzos por lograr el acceso universal a la educación básica y mejorar su calidad y equidad, sin embargo, aún persisten importantes desigualdades educativas en función del origen socioeconómico y de la procedencia cultural, entre la zona rural y urbana y entre escuelas públicas y privadas, lo cual significa que la educación no está siendo capaz, en muchos casos, de romper el círculo vicioso de la pobreza ni de ser un instrumento de movilidad social.

El mayor acceso a la educación ha significado que una mayor diversidad de alumnos acceda a ella, sin embargo, los sistemas educativos siguen ofreciendo respuestas homogéneas que no satisfacen las distintas necesidades y situaciones del alumnado, lo que se refleja en altos índices de repetición y deserción y los bajos niveles de aprendizaje que afectan, en mayor medida, a las poblaciones que están en situación de vulnerabilidad.

Para hacer frente a los altos niveles de exclusión y de discriminación existentes en la mayoría de los países, en los últimos años ha surgido con fuerza el movimiento de inclusión o educación inclusiva. En muchos casos, se está asimilando dicho movimiento al de integración de alumnos con necesidades educativas especiales, cuando se trata de dos enfoques de naturaleza distinta.

El foco de la inclusión es más amplio que el de la integración puesto que su preocupación es hacer efectivo el derecho de todos a una educación de calidad, ya que existen muchos niños y niñas en el mundo, además de aquellos con discapacidad, que no tienen acceso a la educación, o bien reciben una de menor calidad. La educación inclusiva es, antes que nada, un asunto de derechos humanos y un medio para lograr una mayor 
equidad, constituyendo un impulso fundamental para avanzar en la agenda de Educación para Todos.

El foco de atención de la educación inclusiva es la transformación de los sistemas educativos y de las escuelas comunes para dar respuesta a la diversidad del alumnado. A diferencia de la integración, donde el énfasis es la atención de las necesidades específicas de los niños integrados manteniendo inalterables los sistemas educativos, la principal preocupación de la educación inclusiva es transformar las culturas, las prácticas educativas y la organización de las escuelas para atender la diversidad de necesidades educativas del alumnado que son la consecuencia de su procedencia social y cultural y de sus características personales en cuánto a motivaciones, capacidades e intereses. La atención a las necesidades educativas especiales se enmarca en el contex to de la atención a la diversidad de todo el alumnado; ya que todos los niños y todas las niñas, y no sólo quienes presentan alguna discapacidad, tienen diferentes capacidades y necesidades educativas.

La educación inclusiva implica una visión diferente de la educación común basada en la heterogeneidad y no en la homogeneidad, considerando que cada alumno tiene sus propias capacidades, intereses, motivaciones y una experiencia personal única, es decir, las diferencias son inherentes a los seres humanos y, por lo tanto, están dentro de lo normal. Desde esta concepción, el énfasis está en desarrollar una educación que valore y respete las diferencias, viéndolas como una oportunidad para optimizar el desarrollo personal y social y para enriquecer los procesos de enseñanza y aprendizaje.

La atención a la diversidad es una responsabilidad de la educación común y del sistema educativo en su conjunto, por lo que no debe entenderse como una alternativa a la educación actual de los alumnos con necesidades educativas especiales sino como una política de atención a la diversidad, en la que estos alumnos son un elemento importante. En el Informe de la Comisión Delors de UNESCO (cit. en UNESCO, 1997) se invita a adoptar "el respeto por la diversidad" como principio fundamental para "combatir todas las formas de exclusión" en la educación, a fin de devolverle a la educación su "rol central como crisol" que contribuye a la armonía social.

Finalmente, la educación inclusiva es un enfoque diferente para identificar y resolver las dificultades que surgen en las escuelas (Booth y Ainscow, 2004), ya que el problema no es el alumno sino el sistema educativo y la escuela. Desde esta concepción, la preocupación principal es identificar y superar las barreras que enfrentan muchos alumnos y muchas alumnas para acceder a la educación, participar plenamente en las actividades educativas y tener éxito en su aprendizaje. Estas barreras se encuentran en las personas, las políticas, las 
culturas y las prácticas educativas. Hay que tener la convicción de que todos los niños y todas las niñas, sin excepción, pueden aprender y desarrollar las competencias básicas necesarias si tienen oportunidades educativas de calidad y se crean las condiciones para que puedan aprovechar dichas oportunidades.

En la Declaración de la Conferencia Mundial de Salamanca sobre Necesidades Educativas Especiales (UNESCO, 1994), se expresó que las escuelas deben acoger a todos los niños de la comunidad independientemente de sus condiciones personales, culturales o sociales; niños discapacitados y bien dotados, niños de la calle, de minorías étnicas, lingüísticas o culturales, de zonas desfavorecidas o marginales, lo cual plantea un reto importante a los sistemas educativos. Asimismo, se sugiere que las escuelas inclusivas constituyen el medio más eficaz para combatir actitudes discriminatorias, construir una sociedad inclusiva y proporcionar una educación de calidad para todos, mejorando así la eficiencia de los sistemas educativos.

En conclusión, cuánto más inclusivas sean las escuelas comunes en su origen, menos alumnos quedarán fuera de ellas y, por tanto, no será necesario integrarlos a posteriori.

\section{2. ¿PUEDE LA EDUCACIÓN DE LA PRIMERA INFANCIA MARCAR LA DIFERENCIA?}

Actualmente existe una gran evidencia acerca de los enormes beneficios, a corto y largo plazo, que tiene la educación de la primera infancia en el desarrollo de las personas y de las sociedades. Como muy bien sugiere Van der Gaag (2000), el desarrollo temprano de cada niño está relacionado con el desarrollo humano del conjunto de una sociedad o país, por lo que invertir en programas de educación y cuidado de la primera infancia es el comienzo natural de las políticas y programas de desarrollo humano. Para este autor, las dimensiones del desarrollo del niño son las mismas que las del desarmollo humano: salud, incluyendo nutrición, educación, desarmollo social y crecimiento. Incluso, un concepto más amplio de desarrollo humano también incluye otras dimensiones como equidad y los derechos humanos que, como hemos visto anteriormente, son dos elementos claves de una educación de calidad.

La importancia de la educación y cuidado en los primeros años de la vida fue plenamente reconocida en la Conferencia Mundial de Educación para Todos (Jontiem 1990). Esta Conferencia supuso un hito fundamental al reconocer que el aprendizaje y, por tanto, la educación comienzan desde el nacimiento. En su Artículo V se expresa lo siguiente:

“El aprendizaje comienza con el nacimiento. Ello exige el cuidado temprano y la educación inicial de la infancia, lo que puede conseguirse 
mediante medidas destinadas a la familia, a la comunidad 0 a las instituciones, según convenga".

Este reconocimiento constituyó una nueva visión de la educación básica al considerar que ésta comienza en el nacimiento, y no al inicio de la educación primaria como se concebía tradicionalmente. En consecuencia, uno de los objetivos fundamentales del Marco de Acción de Educación Para Todos fue la expansión de la asistencia y de las actividades de desarrollo de la primera infancia, incluidas las intervenciones de la familia y de la comunidad, prestando especial atención a los niños y las niñas en situación de mayor vulnerabilidad, pobres, desasistidos y con discapacidad.

Diez años después, en el Foro Mundial de Educación para Todos (Dakar 2000), se estableció como uno de los objetivos, a alcanzar en 2015, el de expandiry mejorar el cuidado y educación de la primera infancia, especialmente para los niños más vulnerables y con más desventajas. En esta oportunidad, a diferencia de Jontiem, en la formulación del objetivo se contemplan tres aspectos fundamentales que no pueden disociarse si queremos lograr la educación para todos: expansión o acceso, calidad y equidad.

En el ámbito de América Latina, los ministros de educación, reunidos en Cochabamba en el año 2000, también hicieron un reconocimiento sin precedente sobre la importancia de la educación y cuidado en los primeros años de la vida. En la Declaración de dicha reunión (UNESCO 2001), se comprometieron hacia el año 2015 a:

"Incrementar la inversión social en la educación y en el cuidado y protección de la primera infancia, especialmente de la población más vulnerable. Es necesario centrar los esfuerzos en ampliar la oferta educativa para asegurar en los próximos años la universalización del tramo de edad de 3 a 6 años y, progresivamente, ofrecer servicios para los menores de 3 años." (op. cit., Recomendación 29).

No obstante las distintas evidencias y los acuerdos internacionales, el avance de la educación de la primera infancia todavía es lento, especialmente en los países en desarrollo que están más preocupados de alcanzar la universalización de la educación primaria.

En América Latina, en el marco de las Reformas Educativas de los años noventa, muchos países han introducido cambios políticos y estructurales en la educación inicial y/o preescolar. En diez países de América Latina se ha incluido el tramo de 5 años, incluso 4 años en algunos, dentro de la educación básica obligatoria, y se ha incrementado la oferta educativa para los niños de 3 a 5 años. 
La expansión de la cobertura también ha ido en aumento en las últimas décadas aunque todavía es baja en un buen número de países. Si bien existen diferencias significativas entre los países, la cobertura se ha ido incrementando en todos los países, alcanzando un promedio regional del 48\% en el año 2000 (UNESCO, 2003). Cuba es el único país de la región que cuenta con una educación universal para todos los niños y todas las niñas de 0 a 6 años. No obstante, todavía queda mucho camino por recorrer para aumentar la cobertura y mejorar la calidad y equidad en esta etapa educativa.

\subsection{Argumentos para invertir en una educación de calidad en la primera infancia}

Diferentes investigaciones han mostrado que participar o no participar en programas de educación y cuidado de la primera infancia marca la diferencia en el desarrollo de las personas, en la reducción de las desigualdades, en la prevención de alteraciones del desarrollo, en el rendimiento académico posterior, en las oportunidades de empleo y en una mayor productividad.

Incluso diversas investigaciones (Reveco, 2004) han demostrado que un buen rendimiento escolar depende más de la educación temprana que del nivel socio-económico de los niños. Por su parte, algunos autores como Carnoy (2004) y Tedesco (2004) aseguran que las diferentes políticas de equidad que se están desarrollando en América Latina no están teniendo éxito en lograr mejores resultados de aprendizaje en los alumnos; tan sólo una educación preescolar de calidad tiene un impacto importante en los resultados de aprendizaje y en la superación de las desigualdades.

Esto significa que, especialmente en el caso de los niños de familias más pobres, la participación en algún programa 0 actividad de educación y cuidado, aunque no sea de gran calidad, constituye una oportunidad fundamental para tener un ambiente estimulante y las interacciones necesarias que favorezcan su desarrollo y aprendizaje.

Existen al menos cuatro tipos de argumentos para invertir en programas de educación y cuidado de la primera infancia: su importancia para el desarrollo humano y de las sociedades; su positivo impacto en los resultados de aprendizaje y en estudios posteriores; su alto poder para equiparar la igualdad de oportunidades; y su alto retorno educativo. A continuación se desarrolla cada uno de ellos de forma muy sucinta.

\subsubsection{Un adecuado cuidado y educación en los primeros años son determinantes para el bienestar y adecuado crecimiento de los niños y para su futuro desarrollo como persona}

Las evidencias de la investigación en psicología, nutrición y neurociencias indican que los primeros años de vida son críticos en la formación de la 
inteligencia, la personalidad y las conductas sociales. En los tres primeros años de vida tienen lugar grandes conquistas del ser humano, como por ejemplo, el lenguaje y la deambulación; y el desarrollo neuronal y la plasticidad del cerebro son impresionantes.

La educación en los primeros años sienta las bases del desarrollo futuro de las personas en los ámbitos físico, emocional, cognitivo y social. Por ello, los programas han de proporcionar cuidados para la salud y una nutrición adecuada, y una estimulación e intencionalidad educativa que favorezcan el desarrollo integral de todo tipo de capacidades, especialmente las de tipo emocional.

En estas edades, más que en ninguna otra, la influencia y dedicación de los padres son mayores, por lo que es necesaria la participación y educación de éstos de manera que contribuyan de forma efectiva al desarrollo y bienestar de sus hijos. Asimismo, es importante realizar acciones orientadas a sensibilizar a las familias, especialmente de menores recursos, respecto a la importancia de la educación en estos años para que lleven a sus hijos a los diferentes programas. Los datos disponibles muestran que las madres que tienen educación terciaria envían más a sus hijos a los programas o instituciones educativas que quienes tienen educación primaria incompleta (UNESCO, 2004).

\subsubsection{Los programas de educación y cuidado de la primera infancia favorecen los logros de aprendizaje y el desarrollo educativo posterior}

Diferentes estudios han mostrado que los niños y las niñas que participan en programas de la primera infancia tienen mejores logros de aprendizaje en la educación primaria, y repiten y desertan menos que aquellos que no tienen la oportunidad de acceder a ellos.

En una investigación longitudinal realizada por Berrueta y Clement (1994, cit. en UNESCO, 2004), en la que se hizo un seguimiento durante 19 años a niños de niveles socioeconómicos bajos, con y sin asistencia a programas de educación de la primera infancia, se concluyó que aquellos que participaron en un programa educativo de calidad obtuvieron mejores calificaciones, y tuvieron menores niveles de repetición durante toda su educación -básica y media- que aquellos que no asistieron. El $67 \%$ de aquellos que participaron en algún programa se graduó de la educación secundaria versus un $49 \%$ del grupo control.

En América Latina, en un estudio comparativo sobre aprendizajes en lenguaje y matemáticas y factores asociados (UNESCO, 1997, 1998 y 2000), Cuba fue el país que tuvo mejores resultados, tanto en la zona rural como 
urbana. Si bien la explicación de estos resultados depende de un conjunto de variables, es importante señalar que Cuba es también el país de la región que cuenta con mayores tasas de atención en los menores de 6 años.

Finalmente, en una reciente investigación realizada en Chile en que se compara a niños que asistieron a la Educación Parvularia y quienes no lo hicieron con los resultados de la Prueba SIMCE (Sistema de Medición de la Calidad de la Educación) aplicada al 8ํaño básico, se concluye que existe una asociación estadísticamente significativa entre nivel alcanzado en Castellano y en Matemáticas y la asistencia o no a Jardín Infantil. Los(as) niños(as) que lo hicieron obtienen en promedio un mejor resultado en ambas asignaturas que los(as) niños(as) que no lo hicieron". (Reveco y Mella, 1999)

No obstante las evidencias señaladas, es posible constatar que los niños que tienen menores niveles de aprendizaje en la educación básica son los hijos de las familias más pobres, que a su vez son quienes menos acceden a los programas de la primera infancia, ya que la cobertura se concentra en los estratos socioeconómicos medios y altos y en la zona urbana.

\subsubsection{Los programas de educación y cuidado de la primera infancia tienen un alto retorno económico e implican un subsidio directo a las familias más pobres}

Diferentes autores han resaltado la importancia de invertir en capital humano, en las habilidades y conocimientos de las personas, para lograr un desarrollo económico. Concretamente, Robert Fogel, Premio Nobel de economía en 1993, señala que la calidad del desarrollo de la primera infancia tiene un efecto significativo en la calidad de la población (cit. en Young, 2000).

Las alteraciones en el desarrollo durante los primeros años, el bajo rendimiento en la escolaridad posterior, la repetición y la deserción suponen, además del costo personal para los alumnos, un gran costo para los gobiemos. Se estima que el costo de la repetición en los países de la región es de alrededor de siete mil quinientos millones de dólares anuales (UNESCO y Secretaría de Educación Pública de México, 2005).

En la medida que se considere el gran poder preventivo de la educación temprana en problemas como la violencia, drogadicción y delincuencia, sus beneficios pueden traducirse no sólo en términos sociales sino también económicos. La investigación ya citada de Bemueta y Clement muestra que las ganancias de invertir en los programas de la primera infancia equivalen a 7 veces lo invertido, y aproximadamente seis veces en lo que se refiere al bienestar social.

En el caso de las familias más pobres, la educación de la primera infancia constituye un subsidio directo ya que posibilita el acceso de las madres al 
mundo laboral generando ingresos que se gastan en gran parte en los hogares, lo que repercute en una mejor calidad de vida de sus hijos (UNESCO, 2004).

\subsubsection{La educación y cuidado de la primera infancia contribuye a reducir las desigualdades de origen de los niños y las niñas}

La igualdad de oportunidades ha de empezar desde el nacimiento para superar o reducir lo más tempranamente posible las situaciones de desigualdad 0 vulnerabilidad en las que se encuentran millones de niños y niñas en el mundo. La inversión en programas de educación y cuidado de la primera infancia puede contribuir de forma importante a una mayor igualdad social y, por tanto, al desarrollo humano.

A pesar de los esfuerzos realizados por aumentar la cobertura y focalizar la atención en las zonas rurales y en los grupos de mayor vulnerabilidad, existe un problema importante de desigualdad en el acceso y en la calidad de los programas que se ofrecen a los colectivos en situación de mayor vulnerabilidad. En efecto, aunque no se dispone de información suficiente y desagregada, es posible afirmar que actualmente la educación de la primera infancia no llega a quienes más la necesitan para superar su situación de desigualdad. Tampoco se dispone de información suficiente sobre la calidad de los programas que se dirigen a estos grupos, por lo que no es posible determinar si los programas existentes logran el objetivo de superar la situación de desventaja de estos niños y niñas, ni si favorecen su plena participación, desarrollo y aprendizaje.

A continuación se describe cual es la situación de América Latina en relación con la equidad en la educación y cuidado de la primera infancia, que es muy coincidente, por otra parte, con la situación a nivel mundial:

- La cobertura se concentra en los sectores urbanos y en los estratos socioeconómicos medios y altos. La desigualdad en función del origen socioeconómico es la más aguda; incluso en los países con mayor nivel de cobertura, los niños y las niñas que pertenecen a los estratos socioeconómicos de menores ingresos están más excluidos(as) de los programas. Esta situación de desigualdad se agrava por el hecho de que la pobreza va unida a otras situaciones de vulnerabilidad como vivir en zona rural 0 aislada y la pertenencia a pueblos originarios.

En Chile, por ejemplo, la distribución del acceso a programas de educación en la primera infancia es muy desigual en función del origen socioeconómico de los niños y las niñas. El 45,1\% de los niños de 0 a 5 años, cuyas familias pertenecen al 20\% más rico de la población, tiene acceso a algún programa 0 institución educativa, mientras que sólo accede a dichos servicios el 20,7\% del 20\% más pobre de la 
población (UNESCO, 2003). Es decir, los niños más ricos tienen más del doble de oportunidades educativas que los más pobres.

En lo que se refiere a la zona rural, si bien ha aumentado la cobertura en todos los países, sigue habiendo una brecha importante entre la zona rural y la urbana. En Chile, por ejemplo, a pesar de las políticas de focalización, a partir de los años noventa, las tasas de cobertura en zonas urbanas duplican los valores referidos a las zonas rurales, con iguales niveles de matrícula entre géneros en ambos casos (op. cit.).

- No existen problemas de equidad en función del género en cuanto al acceso a los programas de la primera infancia, ya que la paridad entre sexos aparece constante, o en ocasiones levemente a favor de las niñas. Sin embargo, no se descarta la discriminación de las niñas en los ámbitos rurales, indígenas y urbano-marginales.

- En los países que cuentan con información respecto al acceso de niños y niñas de pueblos originarios, se observa que éste es mucho menor que el de los grupos no indígenas. No obstante, en algunos países se han producido algunos avances porque este colectivo ha sido considerado como grupo de atención prioritaria en las políticas.

- Los niños con discapacidad son los más excluidos, a pesar de que algunos países establecen como prioridad en sus políticas la atención de este colectivo. Dada la importancia de una atención oportuna para compensar las alteraciones del desarrollo, es urgente contar con datos desagregados que nos permitan identificar cuántos niños y niñas tienen algún tipo de discapacidad o pueden estar en riesgo de presentarla. Un avance importante al respecto es el desarrollo de políticas de integración dentro de los programas y servicios comunes que se ofrecen a toda la población.

- La cobertura se concentra en las edades más cercanas al inicio de la educación obligatoria. Considerando que los tres primeros años de vida son críticos en el desarrollo de las personas, es importante ampliar la cobertura en estas edades para prevenir alteraciones del desarrollo y para atender tempranamente aquellas que ya hayan hecho aparición.

\section{Calidad de los programas de educación y cuidado de la primera infancia}

Al igual que ocurre en la educación primaria o secundaria, no existe un acuerdo respecto a la definición de una educación de calidad en los primeros años de la vida, ni se dispone de información comparativa a nivel internacional que permita conocer la calidad de los programas y servicios que se ofrecen 
a los menores de 6 años. La multiplicidad de necesidades que es preciso atender en esta etapa, la diversidad de programas formales y no formales y la intervención de distintos sectores y actores, hacen que la definición de calidad sea aún más compleja y relativa que en etapas educativas posteriores.

La complejidad mencionada puede explicar por qué la mayoría de los estudios está más orientada a evaluar las repercusiones que tiene la asistencia a los programas de la primera infancia en la educación primaria 0 en el desarrollo de los niños, que a evaluar la calidad de la educación que se brinda en los mismos.

En los últimos años se están desarrollando indicadores e instrumentos para evaluar la calidad de los programas y servicios, aunque en muchos casos no se está llevando a cabo un debate respecto a qué se considera una educación de calidad en los primeros años de la vida. Al respecto, es preocupante, por ejemplo, la exportación de modelos e instrumentos de la educación básica. Estos suelen estar restringidos a la formulación de estándares en ciertas áreas de aprendizaje, especialmente matemáticas y lenguaje, dejando fuera otros aspectos fundamentales para la supervivencia y el desarrollo integral de los menores.

En los primeros años, la satisfacción de ciertas necesidades de cuidado y protección y el desarrollo de capacidades cognitivas, motoras, sociales, y sobre todo emocionales son cruciales para el crecimiento y el bienestar de los niños y las niñas. Por otro lado, los resultados que alcanzan en estas edades están más influenciados que en otras por las pautas de crianza y la calidad de las acciones de las familias. Lo anteriormente señalado significa que es preciso desarrollar modelos e instrumentos acordes a la naturaleza e idiosincrasia de esta etapa educativa.

Algunos de los indicadores más utilizados para la evaluación de la calidad de los programas de educación y cuidado de la primera infancia suelen ser los siguientes: entorno físico, formación y nivel de calificación de los docentes; relación entre el número de niños y el educador; claridad de los objetivos del programa; características de la gestión y organización de los servicios y los procesos educativos (UNESCO, 2005).

En los países de América Latina, existe una preocupación por la calidad de los denominados "programas no formales", ya que no existen suficientes estudios sobre los procesos, resultados e impacto de muchos de ellos. Esto es especialmente importante, ya que estos programas suelen dirigirse a los niños y niñas en situación de mayor vulnerabilidad, perpetuándose el círculo vicioso de la desigualdad; educación de pobres para pobres.

Los criterios señalados al inicio de este artículo para definir una educación de calidad -derechos, equidad, pertinencia y relevancia- son válidos también 
para la educación de la primera infancia, ya que en ella se sientan las bases de una educación de calidad que promueva el pleno desarrollo, aprendizaje y participación de las personas. Sin embargo, es importante señalar algunos aspectos específicos que es preciso asegurar en esta etapa por las necesidades y las caractenísticas de los niños y las niñas menores de 6 años.

\subsection{La atención integral de necesidades de desarrollo, cuidado y protección}

Como ya se ha señalado, en los primeros años de la vida, además del desarrollo integral de distintas capacidades, las necesidades de cuidado y protección son fundamentales para el desarrollo mismo y el bienestar del niño. El desarrollo cognitivo puede verse claramente limitado si el niño no tiene una alimentación adecuada y un ambiente afectivo y seguro.

Difícilmente un solo programa puede dar respuesta al conjunto de todas las necesidades, por lo que es fundamental establecer estrategias de coordinación intersectorial, entre diferentes instancias del ámbito de la salud o bienestar social, por ejemplo.

\subsection{Ambiente de aprendizaje que de seguridad y afecto a los niños}

En esta etapa de la vida, más que en ninguna otra, es necesario crear entornos afectivos y emocionalmente seguros para que el desarrollo y el aprendizaje tengan lugar. Los niños más pequeños necesitan establecer relaciones afectivas, sentirse valorados y queridos para construir una autoimagen positiva de si mismos y para asegurar su autoestima y bienestar.

Diferentes estudios y autores han señalado la estrecha relación entre el rendimiento y la autoestima con una percepción positiva del clima escolar por parte de los alumnos. Para desarrollar climas positivos, es importante reconocer a cada niño como una persona única, valorando a cada uno por lo que es, teniendo altas expectativas y brindando apoyo a todos los niños y todas las niñas según sus necesidades.

\subsection{Una pedagogía centrada en los niños que considere las diferencias sociales, culturales e individuales en los procesos de aprendizaje}

Como seres humanos tenemos una serie de características que nos asemejan y otras que nos hacen ser diferentes. El origen social y cultural y las características individuales en cuanto a experiencias personales, capacidades, intereses, hacen que no haya dos niños idénticos y que el proceso de aprendizaje sea único e irrepetible en cada caso. De hecho, muchos niños y niñas enfrentan barreras en su aprendizaje y participación debido a la homogeneidad de la oferta y respuesta educativa que caracteriza a nuestros sistemas educativos. 
Una enseñanza centrada en el niño significa construir a partir del conocimiento que tiene, el cual está mediatizado por la cultura y el grupo social a los que pertenece. Supone que el docente descentre la mirada de si mismo para ponerla en el alumno, lo que significa aprender a reconocerlo, respetarlo, entenderlo y, para Maturana y Morin, amarlo (Sancho, 2002).

Ajustar el proceso de enseñanza a las necesidades, competencias y estilo de aprendizaje de cada niño significa ofrecer una variedad de situaciones que sea significativa para todos y no sólo para una minoría. Para ello es preciso conocer bien a los niños; cuales son sus experiencias y conocimientos previos, sus intereses y sus competencias. Lograr que cada niño participe y aprenda requiere utilizar una variedad de estrategias, especialmente las de aprendizaje cooperativo, ya que tienen efectos positivos no sólo en el aprendizaje sino también en la autoestima, las relaciones sociales y el desarrollo personal.

En el caso de los más pequeños, el juego es fundamental para su desarrollo y aprendizaje, ya que a través de él adquieren numerosos conocimientos del mundo físico y social y sobre ellos mismos. También es importante promover situaciones y contextos de aprendizaje que faciliten la acción, experimentación, el juego y el intercambio social con adultos e iguales.

\subsection{La integración de la familia al proceso educativo}

La influencia de las familias en estas edades es aún más decisiva que en otras, por lo que es preciso integrar a los padres para que colaboren con las educadoras y otros profesionales en los procesos educativos. La participación de las familias puede tener niveles muy diversos; desde la aportación 0 elaboración de materiales, a la toma de decisiones en los proyectos 0 decisiones que afectan a sus hijos, pasando por la colaboración en actividades dentro o fuera de las instituciones educativas.

En esta etapa los niños y las niñas han de ampliar sus ámbitos de experiencia y realizar determinados aprendizajes que les permitan resolver las situaciones de la vida cotidiana de manera autónoma. Por ello, es importante fortalecer la articulación entre los distintos ambientes y contextos en los que se desarrolla el niño -familia, comunidad y escuela- con el fin de fortalecer, apoyar y contextualizar los aprendizajes.

La participación de los padres no sólo favorece una mayor coherencia entre el hogary la institución o programa educativo, sino que mejora la calidad de las relaciones con sus hijos y les permite tener un mayor conocimiento de los mismos. El trabajo colaborativo con los padres requiere que las educadoras y los profesionales reconozcan y valoren los conocimientos de las familias, ya que los padres conocen muy bien a sus hijos y pueden aportar dicho conocimiento para optimizar los procesos educativos. 


\section{Desafíos para avanzar hacia una educación de la primera infancia de calidad ${ }^{1}$}

Son varios los desafíos que es preciso enfrentar para avanzar hacia una educación de mayor calidad en la primera infancia. Junto con aumentar la cobertura, especialmente para aquellos que estén en situación de vulnerabilidad, hay que emprender diferentes acciones orientadas a mejorar la calidad, entre las que cabe destacar las siguientes:

\subsection{Aumentar la cobertura, especialmente para aquellos que están en situación de vulnerabilidad}

En el horizonte de los próximos 15 años se debenía asegurar una oferta educativa suficiente para toda la población infantil de 3 a 5 años, especialmente para aquellos que se encuentran en situación de mayor vulnerabilidad. Para ello, es imprescindible ofrecer una diversidad de modalidades de atención, reforzando la calidad de las denominadas no formales, ya que son éstas las que pueden lograr una mayor cobertura y las que tienen una mayor flexibilidad para atender situaciones y necesidades diferenciadas. Es preciso también buscar alternativas diversas para llegar a poblaciones aisladas y marginadas, utilizando en mayor medida los medios tecnológicos de comunicación y de información.

La población de los niños con discapacidad, que presenta un alto porcentaje sin ningún tipo de atención, ha de constituir un objetivo importante dentro de las politicas de la primera infancia, favoreciendo su integración dentro de los programas que existen para toda la población, y proporcionándoles las ayudas y servicios que requieran para atender sus necesidades específicas. En relación con los niños de otras etnias y culturas, ha de tenerse en cuenta la dimensión intercultural y bilingüe en su proceso de aprendizaje.

Lograr una mayor cobertura y equidad implica aumentar de forma sostenida la inversión y desarrollar estrategias de afirmación positiva a la hora de asignar los recursos humanos, financieros y materiales, favoreciendo la atención de los grupos en situación de mayor vulnerabilidad.

La labor del Estado en la atención de los menores de 4 años y de los grupos en situación de vulnerabilidad debería ser más intensa, desde el punto de vista de los recursos, la gestión, los soportes técnicos y la profesionalización de los recursos humanos. Esta mayor participación del Estado ha de realizarse conjuntamente con la acción de la sociedad civil. 


\subsection{Desarrollo de curricula que orienten la atención y desarrollo integral de los niños y las niñas durante toda la etapa educativa, considerando su propia identidad}

Algunos países, dentro de sus procesos de reforma educativa, han elaborado 0 están elaborando curricula para los niños menores de 6 años, aunque en general, sólo abarcan el tramo más cercano al inicio de la educación primaria.

El desarrollo de curricula para este nivel educativo es un aspecto importante para avanzar hacia una mayor calidad, porque muchos de los programas se caracterizan más por su carácter asistencial y de cuidado que por la intencionalidad educativa. Es preciso, sin embargo, que una mayor intencionalidad no se interprete como una mera preparación a la educación primaria. Hay que lograr un equilibrio entre la atención a las necesidades de salud, nutrición, bienestar, protección de los derechos, y el desarrollo psicosocial de los niños.

Los curricula en esta etapa han de ser sumamente abiertos y flexibles para adecuarlos a las diferencias de los niños y de los contextos en los que se desenvuelven, pero también han de considerar el desarrollo de todo tipo de capacidades; cognitivas, motoras, sociales y emocionales.

La educación ha de tener un enfoque de derechos humanos y una visión intercultural de forma que los aprendizajes sean significativos y pertinentes para todos y no sólo para aquellos de las clases y culturas predominantes.

\subsection{Fortalecer la coordinación inter e intrasectorial para atender de forma integral todas las necesidades de los niños y las niñas}

La atención integral de las necesidades de desarrollo, aprendizaje, supervivencia y protección de los niños y las niñas, no significa que cada programa 0 institución ofrezca todos los servicios, sino establecer estrategias de articulación y colaboración entre distintas instituciones o servicios de la comunidad: salud, nutrición, educación, trabajo, justicia y bienestar social. Un problema importante en la mayonía de los paises es la falta de coordinación interinstitucional e intersectorial, por lo que es preciso buscar estrategias que permitan la articulación e integración real de distintas acciones y servicios desde un marco conceptual común, y con funciones y servicios complementarios.

En el sector educativo es fundamental la articulación entre educación inicial y primaria y otras etapas del sistema educativo La alfabetización y educación de adultos es esencial para potenciar el desarrollo y la educación de los niños, por lo que hay que establecer estrategias de articulación entre los programas de educación infantil y los de adultos, en beneficio del desarrollo de la comunidad. 


\section{4. Formación de recursos humanos; docentes, familias y otros profesionales}

Las políticas para mejorar la calidad de la educación basadas en reformas estructurales y en insumos se han mostrado insuficientes para promover cambios significativos. Por otra parte el desarrollo humano, como se ha visto anteriormente, requiere invertir en las personas, en promover sus habilidades y conocimientos. En definitiva, una política que pasa por promover cambios en las personas.

Según el informe de monitoreo de Educación para Todos 2005, la calidad de los programas de Educación y Cuidado de la Primera Infancia es escasa en muchos países debido al bajo nivel de calificación del personal. De un total de 69 países con información disponible, sólo en un 20\% todos los docentes están formados, mientras que en un 75\% la proporción de docentes no formados es mucho mayor que en la enseñanza primaria (UNESCO, 2005). Esta afirmación es consistente con los resultados encontrados en investigaciones realizadas en Estados Unidos (Love, Schochet y Meckstroth, 2000), en las que se enfatiza que las interacciones entre los docentes y los niños y la calidad del personal que los atiende constituyen dos factores fundamentales para lograr resultados de calidad. Los hallazgos encontrados permitieron concluir que la calidad depende de los siguientes factores:

- educación de los docentes con formación especializada en educación temprana,

- formación en servicio más allá de la educación formal,

- experiencia de los docentes con los niños,

- continuidad del personal y que éste se sienta bien con sus condiciones en el trabajo,

- director con la experiencia y formación necesarias para apoyar al personal,

- relaciones con la comunidad, especialmente con aquellas instituciones que puedan proporcionar servicios de salud y otros apoyos,

- espacio físico y seguro.

La preparación de los diferentes recursos humanos que atienden a la primera infancia, es uno de los mayores desafíos que enfrentan los países y requiere de medidas en la formación inicial y en servicio. La variedad de actores y profesionales que intervienen en estas edades, hace necesaria una oferta de formación amplia y diversificada, en la que se aseguren unos conocimientos y estrategias básicas que han de ser comunes para todos, y otros diferenciados en función del rol y el tipo de atención que brinda cada uno. Es importante prestar especial atención a la formación de las madres 0 voluntarios comunitarios que desarrollan programas no formales, elaborando materiales sencillos y pertinentes que les sirvan de apoyo a su labor. 
En cuánto a las familias, está suficientemente demostrado que un mayor nivel de formación y escolaridad de los padres facilita el desarrollo y aprendizaje de los niños, aunque no es tan determinante el capital cultural de éstos, sino la forma en que lo trasmiten a sus hijos. Las expectativas de los padres, la dedicación a sus hijos y la comunicación que establecen con ellos son factores determinantes.

El desarrollo de acciones dirigidas a lograr una mayor preparación de los padres, especialmente los de menores recursos, es un elemento de especial importancia para avanzar hacia una educación de mayor calidad. Estas acciones han de orientarse a fortalecer su rol como primeros educadores de sus hijos, de forma que puedan exigir derechos y asuman sus responsabilidades, participen en la toma de decisiones que afectan a sus hijos y en las actividades educativas que se llevan a cabo en los programas. Las acciones de los padres también han de tener como foco aumentar su nivel de escolaridad, por lo que es muy importante la oferta de educación básica y secundaria para adultos, en la que se incluyan contenidos y módulos relacionados con el papel de los padres y la crianza y educación de los hijos. La utilización de los medios de comunicación de masas puede ser una estrategia muy eficaz para lograr una mayor cobertura en la formación de los padres.

\subsection{Sistemas de monitoreo y de información}

Es urgente contar con un sistema de información amplio que sirva para la toma de decisiones, la provisión y planificación de recursos y de servicios y la mejora de la calidad de los programas. Para ello es preciso el desarrollo de sistemas de información integrada que provean de estadísticas e indicadores, que incorporen los resultados de la investigación educativa y de las evaluaciones de los programas, y que aporten información sobre innovaciones en este nivel educativo.

Se requiere contar con indicadores de acceso a los diferentes tipos de programas de la primera infancia desglosados por edad, género, zona geográfica, y colectivos en situación de mayor vulnerabilidad, de forma que se pueda valorar el nivel de equidad, así como realizar estudios sobre la oferta y la demanda.

Es urgente, asimismo, construir modelos e instrumentos para evaluar la calidad de la educación, lo cual significa, a su vez, construir indicadores que permitan evaluar el desarrollo integral y el aprendizaje de los niños y las 
niñas, la satisfacción de sus derechos, la pertinencia y relevancia de la educación, y el impacto de la familia y el contexto educativo en el desarrollo de los niños y las niñas.

Finalmente, es preciso desarrollar mecanismos de difusión de información y de conocimientos para que lleguen a los distintos involucrados en el cuidado y la educación de los menores y puedan usarlos en la toma de decisiones. Si bien es cierto que existen carencias importantes de información, no es menos cierto que muchas veces ésta no se utiliza para la toma de decisiones.

\section{NOTAS}

1/ Este apartado está basado en gran parte en Blanco, R. (2003). Desafíos de la educación y cuidado de la primera infancia en los países de América Latina y el Caribe. En: II Memorias del Congreso Mundial de Educación Inicial: por una educación de calidad.

\section{REFERENCIAS BIBLIOGRÁFICAS}

Blanco, R., 2003

Blanco, R., 2005

Booth, T. y M. Ainscow, 2004

Carnoy, M., 2004

Hevia, R. y C. Hirmas, 2005

Love, J. M., P Schochet \& A. L. Meckstroth, 2000

Mantovani, J., 1957

MINEDUC, 2004
Desafíos de la educación y cuidado de la primera infancia en los países de América Latina y el Caribe. En: II Memorias del Congreso Mundial de Educación Inicial: por una educacion de calidad. Asociación de Educadores de América Latina y el Caribe. Caracas. pp. 266-297.

Los docentes y el desarrollo de escuelas inclusivas. En: Revista PREALC. Edición monográfica. Protagonismo docente en el cambio educativo. UNESCO/OREALC, Santiago. 2(1) : 174-177.

Índice de inclusión: desarrollando el aprendizaje y la participación en las escuelas. OREALC/UNESCO. Santiago, Chile, $117 \mathrm{pp}$.

La búsqueda de la igualdad a través de las políticas educativas: alcances y límites. En: Seminario de Políticas Públicas de Equidad. Organizado por Fundación Ford, Universidad Padre Hurtado, UNICEF y UNESCO. Santiago.

Políticas de atención a la diversidad cultural. UNESCO/ OREALC, Santiago de Chile. En prensa.

Investing in effective childcare. Lessons from research. En: Mary Eming Young (ed.). From early child development to human development: investing in our children's future. The World Bank. Washington. pp. 145-193.

Educación y plenitud humana. Ateneo. Buenos Aires.

Nueva visión y perspectiva de la educación especial: Informe de la comisión de expertos. Ministerio de Educación de Chile. Santiago. 
Reveco, 0. y O. Mella, 1999

Reveco, 0., 2004

Sancho, J. M., 2002

\section{Secretaría de Educación Pública de México y UNESCO, 2005}

Tedesco, J. C., 2004

UNESC0, 1990

UNESC0, 1994

UNESCO, 1997

UNESCO, 1997, 1998, 2000

UNESC0, 2000

UNESC0, 2001

UNESC0, 2003

UNESCO, 2005

Van der Gaag, J., 2000

Young, M. E., 2000
Impacto de la Educación Parvularia en la Educación Básica. Serie Documentos de Estudios. N ${ }^{\circ}$. JUNJI. Santiago.

Participación de las familias en la educación infantil latinoamericana. UNESCO/OREALC, Santiago.

A propósito de los factores que promueven la mejora de la enseñanza. En: La mejora de la escuela. CIDE/ Ministerio de Educación, Cultura y Deporte de España y Ediciones Octaedro. Madrid.

Panorama educativo 2005: progresando hacia las metas. Informe preliminar. Proyecto Regional de Indicadores Educativos de la Cumbre de las Américas. UNESCO/ OREALC. Santiago.

Igualdad de oportunidades y política educativa. En: Seminario de Políticas Públicas de Equidad. Organizado por la Fundación Ford, Universidad Padre Hurtado, UNICEF y UNESCO. Santiago.

Conferencia Mundial de Educación para Todos. Jontiem, Tailandia. UNESCO/Paris.

Conferencia Mundial de Salamanca sobre Necesidades Educativas Especiales: Acceso y calidad especiales. Salamanca, España.

Learning: The Treasure within. Correo de la UNESCO, México.

Primer estudio internacional comparativo de lenguaje, matemática y factores asociados en 13 países de América Latina. Primer Informe 1997, Segundo Informe 1998, Informe Técnico 2000. UNESCO/OREALC, Santiago.

Foro Mundial de Educación para Todos, Dakar, Senegal. UNESCO/París.

Declaración de Cochabamba y recomendaciones sobre políticas educativas al inicio del siglo XXI. Cochabamba, Bolivia, Marzo 2001. UNESCO/ OREALC, Santiago.

Educación para Todos en América Latina: un objetivo a nuestro alcance. Informe regional de monitoreo de EPT. UNESCO/OREALC, Santiago.

Informe de seguimiento de la Educación para Todos en el mundo. El imperativo de la calidad. UNESCO/ París.

From early child development to human development. En: Mary Eming Young (ed.). From early child development to human development: investing in our children'sfuture. The World Bank. Washington. pp. $63-78$.

Introduction and Overview. En: Mary Eming Young (ed.). From early child development to human development: investing in our children's future. The World Bank. Washington. 